\title{
Role of Nutrients Competition in Immunometabolism - Effects on Immune Responses - A Systematic Review
}

\author{
Kulvinder Kochar Kaur ${ }^{1 *}$, Gautam Allahbadia ${ }^{2}$ and Mandeep Singh ${ }^{3}$ \\ ${ }^{1}$ Scientific Director, Dr Kulvinder Kaur Centre for Human Reproduction, Jalandhar, Punjab, India \\ ${ }^{2}$ Scientific Director, Rotunda-A Centre for Human Reproduction, Mumbai, India \\ ${ }^{3}$ Consultant Neurologist, Swami Satyanand Hospital, Jalandhar, Punjab, India \\ *Corresponding Author: Kulvinder Kochar Kaur, Scientific Director, Dr Kulvinder Kaur Centre for Human Reproduction, Jalandhar, \\ Punjab, India.
}

Received: August 20, 2019; Published: September 30, 2019

\begin{abstract}
With the alteration in metabolism in the cells, variety of immune subsets get activated. The fuel provided for these changes comes from nutrients like glucose, amino acids, and fatty acids, which are normally associated in a big way with the immune cell fate and function. The newer hypothesis that is being proposed, point that the control of nutrient utilisation via competitive uptake and use is essential for regulating immune responses. The idea of this review is to discuss various immune microenvironments in which limitation of nutrients occurs, and how important availability of nutrients is in the control of immune responses.
\end{abstract}

Keywords: Immune Cells; Nutrient availability; Immunometabolism; Tumours; Infections

\section{Introduction}

In the immune cells very fast and marked changes occur following an immune response with simultaneous changes in cellular metabolism. The demands of nutrients are large as far as nutrients are concerned that include glucose, glutamine and fatty acids, that get metabolized for ATP formation for energy expenditure. Also these nutrients are used in the meantime for the synthesis of cellular components, that include protein, nucleic acids and lipids (Figure 1). Variety of metabolic configurations that is supported by a lot of nutrients have been revealed for immune subsets, that facilitate the specialized immune functions of individual cells [1]. For getting an insight regarding how separate immune cells get affected by immune microenvironments, where there are limited nutrients, one has to understand the various nutrient demands and metabolic configurations of different immune cells, which is described briefly here [1-3]. Further the conditions where nutrients might become a limiting factor within different types of immune microenvironment, that includes the tumour microenvironment and sites of infection is discussed. Ultimately the results of nutrient deprivation on nutrient signaling pathways and its impact on immune function is detailed.

\section{Methods}

Thus we searched the pubmed engine with the MeSH terms "Immunometabolism"; "tumors"; in infections-"all viruses", "bacteria”, in particular immune cells like T Cells (all kinds), BCells, Natural Killer Cells, Myeloid cells and nutrients utilized.

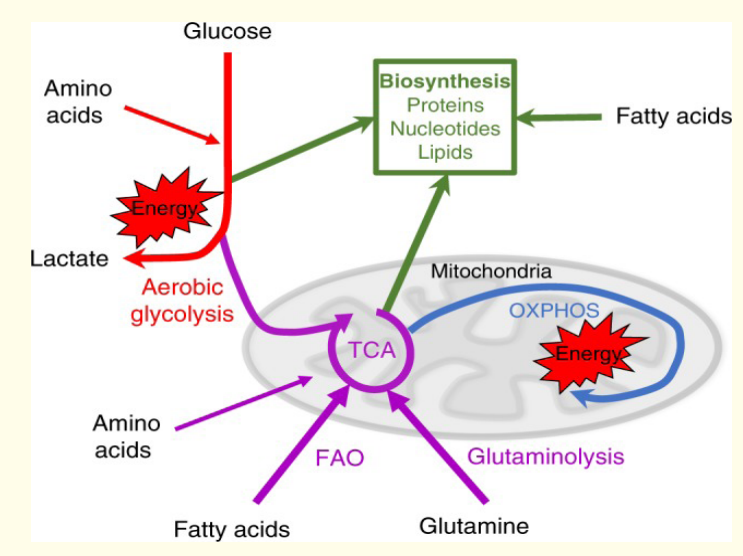

Figure 1: Courtesy ref -50.Metabolism configured to support energy homoeostasis and biosynthesis. Cellular metabolism can be configured to efficiently generate energy in the form of ATP. Glucose is metabolised by aerobic glycolysis (red) and via glycolysis coupled to the tricarboxylic acid (TCA) cycle (purple) to drive oxidative phosphorylation (OXPHOS) (blue) and the generation of energy in the form of ATP. Additional fuels, including fatty acids and the amino acid glutamine, can be used to support OXPHOS. Various other amino acids can also feed into both glycolysis and the TCA cycle. In addition to fuelling energy production, glucose and amino acids can be metabolised and used to support biosynthetic processes (green). Intermediates of glycolysis and the TCA cycle can be diverted into metabolic pathways to generate biosynthetic precursors important for the synthesis of lipids, nucleotides and proteins. Fatty acids can also be directly used for biosynthesis. 


\section{Results}

We found a total of 16840 articles of which we selected 50 articles for this review. No meta-analysis was done.

\section{Metabolic configurations and nutrient demands}

For the fate and functions of immune cells cellular metabolism remains the critical factor. Disrupting metabolic signaling pathways can cause loss of normal immune function or changes in the immune cell differentiation. Since immune cells have a variety of functions, it is not surprising that their nutrient and energy demands are high. Once Tcells differentiate into memory, regulatory and effector Tcell subsets, the nutrient they utilize and the metabolic configurations they use, adjust to match their particular metabolic demands. E.g. is the various metabolic needs of naïve and effector T cells subsets (Figure 2) [4]. The metabolic needs of naïve $\mathrm{T}$ cells are low in view of low metabolic rate and minimal biosynthetic needs when comparing them with activated $\mathrm{T}$ cells subsets, that is in view of relative inactivity. Thus naïve $\mathrm{T}$ cells are consuming small fuel contents like glucose, glutamine and fatty acids, that they metabolize via oxidative phosphorylation (OXPHOS), mainly for obtaining energy (Figure 1 and 2). Rates of metabolism markedly increase in Tcells following immune activation because of proliferative, expansion and the introduction of a variety of effector functions like generation of large quantities of cytokines, which needs remarkable quantities of energy and cellular biosynthesis. This=>an increased demand for nutrients, like glucose and glutamine, along with amino acids like serine and arginine, for fueling both bioenergetic along with biosynthetic pathways (Figure 1 and 2) [4-8]. Effector $T$ cells have > rates of glucose and glutamine uptake, that then get metabolized by aerobic glycolysis in the cytoplasm and the tricarboxylic acid (TCA) cycle in the mitochondria [9]. Further Effector $\mathrm{T}$ cells also have greater uptake of other amino acids that includes leucine, serine and tryptophan besides other amino acids sometimes [6,7]. This metabolic configuration supports the combined cellular requirements of effector $\mathrm{T}$ cells for energy and biosynthesis (Figure 1). In contrast, memory T cells return to a quiescent state and have decreased synthetic demands, and thus go back to using oxidative metabolism for>efficiency energy generation. Also memory $\mathrm{T}$ cells produce intracellular fuel reserves in the form of glycogen and triacylglycerides that gives them metabolic security and plasticity that is necessary to support both longevity along with rapid recall responses which are central to their functions $[4,5]$. The control over Effector $\mathrm{T}$ cells is carried out by Regulatory T cells, which don't have high biosynthetic demands and so mainly employ OXPHOS, that is fueled by external fatty acids that get imported as well as metabolized through a pathway known as fatty acid oxidation to develop energy, (Figure 1) [10]. However when Regulatory T cells do enter cellular division, they move over to glycolytic metabolism, for supporting biosynthetic needs for growth and proliferation [11]. If this cellular metabolism in T cells is disturbed, it causes impaired $\mathrm{T}$ cell function and changes the differentiation of $\mathrm{T}$ cells towards effector, memory or regulatory subsets [3]

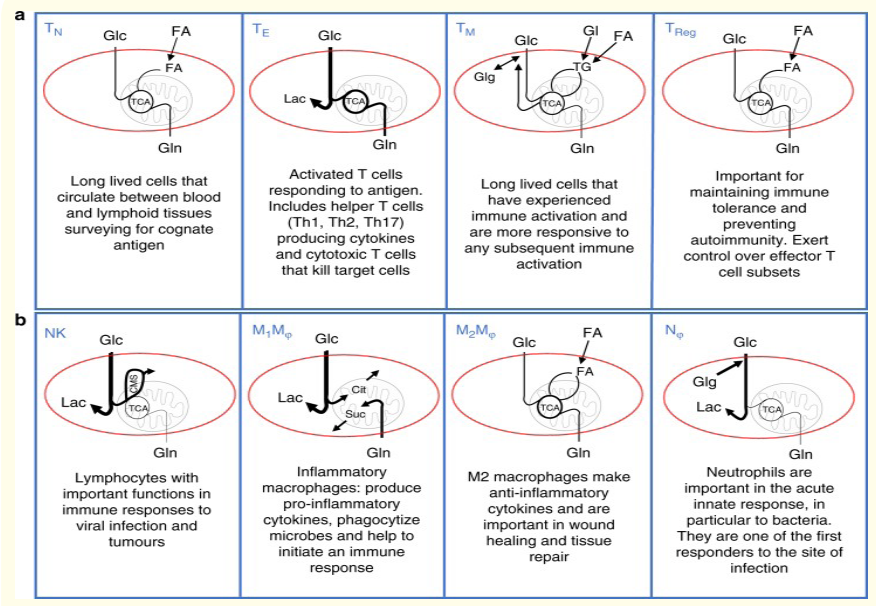

Figure 2: Courtesy ref no-50.Illustrating the different metabolic configurations of immune cells. a T cells: Naive T cells (TN) have low metabolic rates fueled by glucose and glutamine. Effector $\mathrm{T}$ cell (TE) subsets tend to have elevated levels of both aerobic glycolysis (for metabolising glucose to lactate (Lac)) and OXPHOS (as fueled by glucose (Glc) and glutamine (Gln)). Memory T cells (TM) maintain intracellular fuel stores in the form of glycogen (Glg) and triacylglycerides (TG) fueled by glucose and fatty acid (FA) uptake, and primarily use OXPHOS rather than glycolysis. TM have metabolic plasticity as they can engage multiple opposing metabolic pathways including gluconeogenesis/glycolysis, glycogenesis/ glycogenolysis and FA synthesis/FA oxidation. TG stores are generated using imported glycerol (Gl). This metabolic configuration supports two key features of TM cells; long term survival by providing dependable fuel sources within the cell (TG and Glg) and rapid metabolic responses to re-stimulation because the metabolic machinery is already present and in use. Regulatory $\mathrm{T}$ cells $\left(\mathrm{T}_{\text {Reg }}\right)$ import FA for use in biosynthesis and to generate energy through FA oxidation. b Other immune cells: Natural killer (NK) cells primarily use glucose as a fuel, which supports aerobic glycolysis and drives OXPHOS through the citrate-malate shuttle (CMS) but not the TCA cycle. In M1 macrophages (M1M $\varphi$ ) the TCA cycle is broken, and glucose is metabolised to lactate and citrate (Cit) (used to make immunoregulatory molecules such as itaconate) while glutamine is metabolised to succinate (Suc) (used to generate mitochondrial ROS). By contrast, M2 macrophages (M2M $\varphi$ ) maintain an intact TCA and favour oxidative metabolism that is fuelled by the uptake of fatty acids, glutamine and glucose. Neutrophils primarily use glycolysis fuelled by glucose uptake and internal glycogen stores, and have very low OXPHOS.

B lymphocytes/B cells, constitute important cells which produce antibodies. They further upregulate glucose uptake and metabolic genes once B C ell antigen receptor gets activated for fuelling energy generation and biosynthesis: in particular, de novo lipogenesis is needed during the differentiation of plasma cells, that are the ones who generate huge quantities of high affinity antibody 
[12]. Glutamine can also be utilized by B cells, and are especially essential for B Cell survival during hypoxic situations [13]. The other lymphocytes like activated natural killer (NK) cells get fuelled by glucose but not by glutamine (figure 2). In this case glucose simultaneously supports high rates of aerobic glycolysis and mitochondrial respiration, with the latter getting obtained through the engagement of citrate-malate shuttle, instead of the TCA cycle, for driving OXPHOS [14]. Right now it is not known why NK cells utilize this Metabolic configuration but it appears that this is essential for supporting epigenetic control since the citrate-malate shuttle also produces acetyl-CoA, that is the substrate for histone acetylation.

Myeloid cells also can adapt separate metabolic phenotypes and thus have varying needs for nutrients. Inflammatory M1 Macrophages use glycolytic metabolism and mostly shut down OXPHOS; the TCA cycle is not occurring in these cells, and the TCA cycle enzymes are reappointed into 2 linear metabolic pathways, which produce essential immune regulatory molecules, i.e. this Metabolic configuration is necessary for the proinflammatory function of these cells [15]. While M2 macrophages, have a longer life as compared to M1 Macrophages, and are required for wound healing and tissue repair, they maintain an intact TCA Cycle and prefer oxidative metabolism fuelling OXPHOS with glutamine and fatty acids [2]. If metabolism of macrophages is interfered with, then the differentiation of M1 vis a vis M2 Macrophages gets changed so that with the inhibition of glycolysis stimulates the differentiation of M2 macrophages over M1 macrophages [3]. Finally the amino acid arginine is necessary for both M1 and M2 Macrophages as a subs rate for the enzymes inducible nitric oxide synthase (iNOS) and arginase respectively.

Granulocytes like neutrophils have little amounts of OXPHOS and are dependent basically on glycolysis [3]. What is funny is that inflammatory neutrophils possess large glycogen stores which are intracellular fuel depots, which can be utilized to maintain this glycolytic metabolism when glucose is absent [16]. This may offer neutrophils, an advantage in inflammatory microenvironments at a place having limited extracellular glucose levels. Dendritic cells (DC's)have their own glycogen stores, which are necessary for fuelling their immediate glycolytic response after LPS stimulation [17]. Glycolytic prevention can both inhibit and increase DC functions, on the basis of its timing during activation is of interest. If glycolysis is inhibited at the time of initial activation, impaired DC function occurs, and on glycolysis inhibition $8 \mathrm{~h}$ following the earlier activation, a time when most likely DC's have arrived at the draining lymph node, increases DC's pro-inflammatory function gets increased along with induction of T cell responses $[17,18]$.

In toto it has been found that immune cell subsets have variable demands for nutrients, that is specific for the subset, so they will get differentially affected by nutrient restrictive microenvironments like tumors or sites of infection. This favours the theory that nutrient availability could play an essential role in shaping immune response.
Immune microenvironments and availability of nutrients

It has been known for a long time that tumours are highly glycolytic and that they have a marked appetite for glucose, that is used to support unrestrained growth of the tumor and proliferation. The increased glucose utilization by tumor cells rapidly utilizes the glucose that is brought by the blood that=>low extracellular glucose within solid tumors [19]. Some tumors also depend on glutamine to support their energy and biosynthetic demands [20], and there is some evidence that points that glutamine levels become limiting in some tumor microenvironments [21]. These features make the tumor microenvironments a metabolically restrictive setting for infiltrating immune cells, which, as published has an effect on the cancer immunotherapies effectiveness as int he case of human melanoma, tumor cells that possess high glycolytic rates have been found to be resistant to adoptive $\mathrm{T}$ cell therapy showing poor $\mathrm{T}$ cell infiltration and cytotoxicity [22].

Question arises whether same mechanisms that are seen in the tumor microenvironment affect immune cells at other immunological sites? There is proof that competition for nutrients is also applicable at sites of pathogenic infections. Multiple viruses reprogramme the cells they infect for increasing glycolysis $[23,24]$. while some have been documented to enhance glutamine metabolism in cells that they infect [25]. Enhanced glycolysis and/or glutaminolysis are of advantage for the virus, as they give biosynthetic material for construction of new virus particles and complete the viral life cycle. In the Same fashion intracellular bacteria like mycobacterium tuberculosis also reprogramme the host cell for enhanced glucose metabolism and glycolysis [26]. Higher fuel usage by these infected cells is going to cause reduced concentration of extracellular glucose and glutamine in the local microenvironment. Further many extracellular bacteria like Staphylococcus aureus, use glucose as their main fuel source, and lots of bacteria can accumulate at a given site when infection occurs [27]. Decreased glucose levels have been documented in patients suffering from meningitis [28]. Hence it is possible that glucose levels will become reduced at such infection sites, and the greater utilization of glucose and glutamine by bacteria or virally infected cells will influence responding immune cells via reduced nutrient availability in the local Immune microenvironment.

Further besides the competitive utilization of glucose and glutamine, levels of other nutrients can get manipulated within Immune microenvironments. Tumors can exhaust amino acids content like arginine and tryptophan from the tumor microenvironments by expressing catabolic enzymes or by recruiting cells which express these enzymes. Arginine can get used by the enzymes iNOS, that is often expressed in tumor cells [29], along with by arginase, expressed by tumor associated fibroblasts and macrophages (TAM's) [30]. For T and NK cells responses Arginine is necessary and depletion of Arginine in the tumor microenvironment, inhibits antitumor T Cells responses [8,31]. Further, tryptophan gets depleted by the enzyme indoleamine 2, 3 -dioxygenase ((IDO), that is often seen in high amounts in tumor cells or tumor associated cells like 
iatrogenic DC's [30,32]. IDO mediated T cell and NK Cell inhibition is because of a combination of tryptophan depletion and the synthesis of the metabolite kynurenine, that impacts the function of $\mathrm{T}$ and NK Cell, atleast partially, via acting on the aryl hydrocarbon receptor (AhR) [33]. Initially IDO was thought to be an innate mechanism of the host defense against infection [34]. Effects of IDO activity on the local distribution of tryptophan and kynurenine was implicated in the growth inhibition of particular bacteria, parasites and viruses [35]. Activity of IDO at these infection sites will thus have implications for immune cells that include T cell and NK Cells. In the same way for tumours, pathogens too utilize ways to deprive immune cells of arginine as part of their immune evasion methods. E.g. Helicobacter pylori bacteria express arginase to exhaust the local microenvironment of arginine and in doing so prevent iNOS -expressing macrophages from producing anti-microbial nitric oxide (NO) [36]. Even in severe malaria low levels of systemic arginine and NO production is seen [37].

Hence multiple ways are there which can cause glucose exhaustion along with different amino acids in pathological Immune microenvironments, which can cause changes in the immune function and responses to tumours or infection.

What is now known is that tumours and pathogens compete with immune cells for nutrients as a way for dodging immune system strategically, but also there can be competition for nutrients between various immune cells, that might be a normal physiological mechanism to regulate immune responses. Immune situations exist, where immune responses with increased metabolism and nutrient demands compete with each other to use the fuels available, like in inflammatory lymph nodes, in which, a rapid rise in the number of activated immune cells, or within the germinal centre in which there is a concentration of metabolically active B cells and follicular T helper Cells. Best e.g. of competition of nutrients between immune cells might have a role in modulating immune responses is obtained by observing DC-TCell interactions. Antigen presenting DC's might become starved of nutrients, like glucose, in view of competitive nutrient uptake by neighboring cells, especially in activating CD8 T Cells [25]. Glucose deprivation of DC might occur in increased DC proinflammatory outputs, that includes the expression of interleukin 12 and costimulatory molecules that=> increased CD8 $\mathrm{T}$ cell responses [25].

Now it is well accepted that T Lymphocytes increase nutrient uptake following Antigen stimulation via upregulation of the expression of nutrient transporters. This is a crucial step for the production of effector Tcells; T cells that don't have particular glucose or amino acid transporters, do not differentiate into effector Tcells. At the time of activation, CD8 T cells cluster around Antigen presenting DC 's within the lymph nodes [38]. These clustering T cells can exhaust the tumors from the microenvironments surrounding the DC's (figure 3). For corroboration of this cocultures of clustering CD8 $\mathrm{T}$ cells can inactivate the nutrient sensitive mammalian target of Rapamycin Complex 1 (mTORC1) signaling pathways in the interacting DC's [25] (figure 3). Actually Antigen presenting DC 's might be present at the centre of cell clusters made up of various types of activated immune cells with increased nutrient uptake rates, besides CD8 Tcells, including NK Cells, CD4 T Cells and Pcd [39]. Hence one wants to hypothesize that starvation of DC's, that lead to enhancement of DC output, is a physiological mechanism for the regulation of $\mathrm{DC}$-induced $\mathrm{T}$ Cell responses, a setting where nutrients are working as an immunological signal (figure 3). A concept that appears fascinating but requires to be proved.

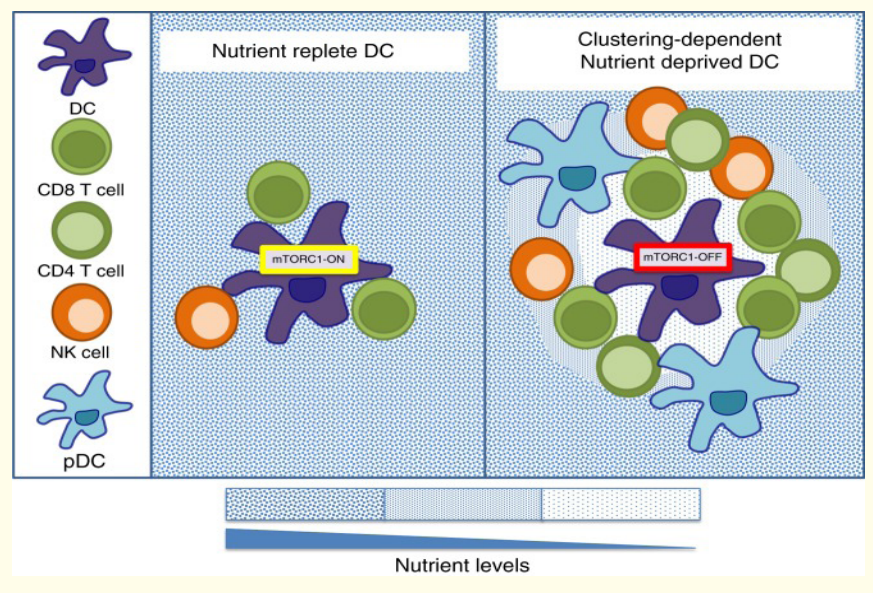

Figure 3: Courtesy ref no-50.Competition for nutrients between immune cells. Antigen-presenting dendritic cells (DC) can be found at the centre of cell clusters consisting of numerous different types of activated immune cells, including CD8 T cells, CD4 T cells, NK cells and plasmacytoid dendritic cells (pDC), with elevated nutrient uptake rates that will compete for nutrients (blue dots). Depending on the number of clustering cells surrounding an antigenpresenting DC, nutrients may be available (left panel) or depleted (right panel) in the immediate surrounding microenvironment due to competitive uptake. Nutrient starvation will have consequences for the DC including the inactivation of mTORC1 signalling, which has been linked to increased proinflammatory DC functions.

Nutrient competiton between $\mathrm{T}$ cells is another proposal regarding the method for $\mathrm{T}$ cell selection, which recognize the antigen with great affinity [40]. As compared with those with low affinity TCR, high affinity TCR -antigen interactions induce a greater robust and sustained metabolic response, with enhanced expression of glucose transporters and glycolytic genes [41]. Hence it has been pointed that high affinity $\mathrm{T}$ cell clones might out compete the low affinity counterparts for nutrients causing nutrient starvation along with apoptosis of these low affinity T cell clones [40]. Hence one can speculate other conditions where neighbouring immune cells might compete for nutrients in the same ways. E.g., during $B$ cell germinal centre responses, a single follicular helper $\mathrm{T}$ cell gets surrounded by a massive number of activating $B$ cells with increased nutrient demands. But the inability to examine nutrient excess at the single cell level is a technical barrier, which right now isa limiting factor regarding any more evaluation of nutrients as important immunological signals. 
How changed nutrient availability affects -signaling and immune outputs

Nutrient restrictive microenvironments will impinge on metabolic pathways on immune cells directly, and besides that will also effect nutrient -sensitive signaling pathways that are necessary for immune regulation. Multiple signaling pathways which are closely related to the control of immune responses can get affected by glucose and glutamine. AMPK represents an indirect glucose sensor which becomes activated once ATP or glycolytic intermediate fructose -1, 6 bisphosphate, level gets reduced in view of decrease in glucose levels [42]. In effector Tcells, AMPK, can get activated within an hour of getting l placed in low amounts of glucose [43]. Glutamine is also essential for ATP synthesis on effector T cells and AMPK can get activated by glucose restriction in these cells [43]. AMPK regulates mTORC negatively, which is an essential regulator of metabolism with multiple effects by which they regulate immune cell functions [43]. (Figure 4). mTORC 1 shapes T cell differentiation, control NK cell differentiation, along with effector

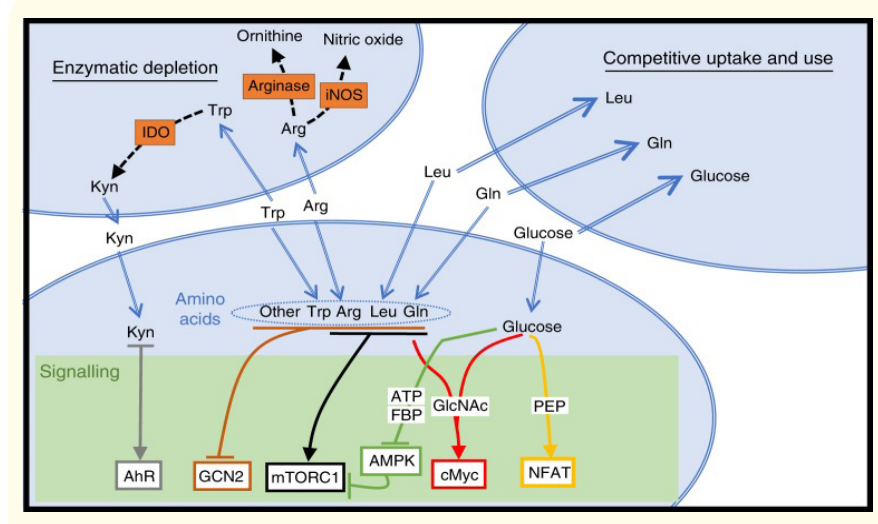

Figure 4: Courtesy ref no.50.Competition for nutrients and the impact on signal transduction. Decreased levels of various nutrients within immune microenvironments could occur due to competitive uptake by surrounding cells. Alternatively, the expression of enzymes that consume nutrients, such as arginase, inducible nitric oxide synthase (iNOS) and Indoleamine-pyrrole 2,3-dioxygenase (IDO), can lead to reduced levels of arginine (Arg) and tryptophan (Trp). Limiting levels of nutrients will affect various signalling pathways. Mammalian target of rapamycin complex 1 (mTORC1) signalling is sensitive to levels of arginine, leucine (Leu) and glutamine (Gln). Glucose deprivation will also activate AMP-activated protein kinase (AMPK) due to reduced levels of ATP or fructose-1,6-bisphosphate (FBP) leading to the inhibition of mTORC1 activity. The metabolite phosphoenolpyruvate (PEP), generated when glucose is metabolised by glycolysis, can affect the duration of NFAT signalling. Gln and glucose are required for the production of uridine diphosphate $\mathrm{N}$-acetylglucosamine (GlcNAc) that is important in sustaining the expression of the transcription factor cMyc. Decreased levels of amino acids in general will lead to the activation of general control nonderepressible 2 (GCN2). The product of IDO-mediated Trp metabolism, kynurenine (Kyn), can promote signalling through the aryl hydrocarbon receptor (AhR).

NFAT nuclear factor of activated T cells. function and control the function of Antigen presenting DC 's (figure 5). Thus changes in AMPK/mTORC1 signaling in view of glucose restriction causes inhibition of inflammatory T celland NK cell responses while promoting $\mathrm{T}_{\text {Reg }}$ differentiation (Figure 5).

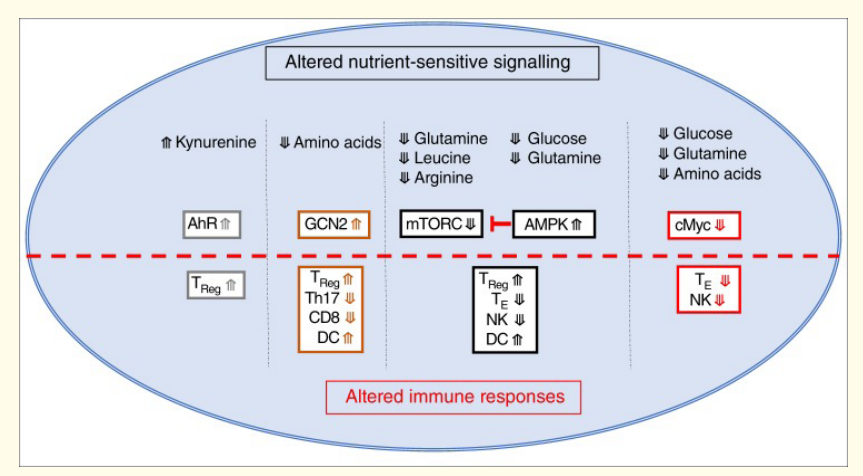

Figure 5: Courtesy ref no 50.Immunological consequences of changes in nutrient signalling. Activation of AMP-activated protein kinase (AMPK) or inhibition of mammalian Target of Rapamycin Complex 1 (mTORC1) signalling promotes the differentiation of regulatory $\mathrm{T}\left(\mathrm{T}_{\mathrm{Reg}}\right)$ cells over effector $\mathrm{T}$ cell subsets (TE), inhibits natural killer (NK) cell functions, and increases the proinflammatory outputs of dendritic cells (DC). Loss of cMyc expression inhibits the functions of TE subsets and NK cells. Activation of general control nonderepressible 2 (GCN2) signalling promotes $\mathrm{T}_{\text {Reg }}$ differentiation, inhibits Th17 differentiation, inhibits CD8 T cell function, and enhances the function of DC. Kynurenine (Kyn)-mediated aryl hydrocarbon receptor (AhR) signalling promotes the differentiation of $\mathrm{T}_{\mathrm{Reg}}$

Fructose -1, 6 biphosphonate does not represent the only glycolytic intermediate which has an effect on immune signaling pathways. Phosphoenolpyruvate (PEP), that is another glucose metabolite, affects $\mathrm{Ca}^{2+}$ signaling and lessnuclear NFAT, ending in defective $\mathrm{T}$ cell activation [19].

Besides fuelling glycolysis and OXPHOS, glucose and glutamine also get utilized for generating uridine diphosphate $\mathrm{N}$ acetylglucosamine (UDP-GlcNAc), the substrate for 0- GlcNAcylation, that is dependent on the supply of both glucose and glutamine in Tcells, pointing that OGT. and O- GlcNAcylation, are essential nutrient sensing mechanisms in these cells [9]. OGT has been documented to be necessary for normal $\mathrm{T}$ cell development, activation and clonal expansion [9]. Mechanically, a lot of signaling molecules which are necessary for $\mathrm{T}$ cell function are found in to be $\mathrm{O}$ - GlcNAcylated, that include-Myc, NFAT, and nuclear factor -KB $[9,44]$. This protein modification has not been investigated deeply in other immune cell subsets.

Besides glutamine, other amino acids regulate various signaling pathways, which are important for immune function. E.g. the activity of mTORC1 is very sensitive to the level of a number of amino acids that are leucine, arginine and glutamine [45]. Further, the transcription factor c-Myc is also controlled by amino acids avail- 
ability. With very short half life of c-Myc protein in lymphocytes and its sustained expression in cells can only be attained in cells which have high rate of amino acid uptake and protein synthesis $[6,9,14,46]$. For the activation and differentiation of $\mathrm{T}$ cell subsets cMyc is critical, besides that of other lymphocytes, that include Bcells and NK cells (figure 5) [14,46,47]. Further the serine/threonine protein kinase General control nondepressible (GCN2) is a direct sensor of low cellular amino acids, and is activated via binding to unchanged transfer RNA (tRNA) (Figure 4) [48]. Action of GCN 2 is related to the function of several immune cells. GCN2 activation in DC's causes increased antigen presentation to CD8 cells. On the other hand GCN2 activity in gut antigen presenting cells prevents increased Th17 responses, with mice who are deficient in GCN2 develop greater Th17 responses and colitis that is of greater severity in an induced colitis model [48]. Tcell responses get repressed by IDO, atleast partially, by depletion of tryptophan amounts, which results in activation of GCN2 within the T cells (figure 4). In CD8 cells GCN2 causes arrest of proliferation and anergy, and on the other hand activation of GCN2 in CD4 T Cells can result in the production of regulatory T Cels (figure 5) [49].

Conclusions and future directions regarding in vivo metabolic analysis

With the help of in vitro and ex vivo metabolic analysis, we have received greater insight on the metabolic phenotype that is adopted by immune cells. Though a lot of information has been passed on by these publications, the metabolic phenotype that has been revealed might not get recapitulated in vivo. It is known that the metabolic phenotype of immune cells rely on the availability of proper fuels like glucose and glutamine, that are definitely available in low quantities in vivo as compared to culture conditions that is utilized in laboratory. The result of limited fuels availability , in vivo within discrete microenvironments will be restriction of metabolc pathways which control the fate of immune cells and their function. Still we lack insight regarding when and where nutrients are available in vivo is markedly hindered by the nonavailability of research tools for measuring nutrient distribution at the single cell level. Hence working out how nutrient supply affects the metabolism, signaling and hence immune cell function in different and complex Immune microenvironments is still a big challenge in the field of immunometabolism.

\section{Bibliography}

1. O'Neill LA., et al. "A guide to immunometabolism for immunologies". Nature Review Immunology 16 (2016): 553-565.

2. Murray PJ., et al. "Snapshot: immunometabolism". Cell Metabolism 22 (2015): 190-191e1.

3. Loftus RM and Finlay DK. "Immunometabolism: Cellular metabolism turns immune regulation'. Journal of Biological Chemistry 291 (2016): 1-10.

4. Back MD., et al. "T Cell metabolism drives immunity". Journal of Experimental Medicine 212 (2015): 1345-1360.
5. Ma R., et al. "A Pck1 directed glycogen metabolic programme regulates formation and maintenance of memory CD8+T Cells". Nature Cell Biology 20 (2018): 21-27.

6. Sinclair LV., et al. "Control of aminoacids transport for arginine transporters coordinates the metabolic reprogramming essential for T Cells differentiation". Nature Immunology 14 (2013): 500-508.

7. Me EH., et al. "Serine is an essential metabolite for effector T Cells expression". Cell Metabolism 25 (2017): 345-357.

8. Geiger R., et al. "L-Arginine modulates T Cells metabolism. And enhances survival and anti- tumor activity". Cell 167 (2016): 829-42. e13.

9. Swamy M., et al. "Glucose and glutamine fuel protein 0GlcNAcylation to control Tcell self renewal and malignancy". Nature Immunology 17 (2016): 712-720.

10. Zeng H and Chi H. "Metabolic control of regulatory T Cells development and function”. Trends Immunology 36 (2015): 3-12.

11. Gerriets VA., et al. "Foxp3 and Toll like receptor signaling balance Treg cell anabolic metabolism for suppression". Nature Immunology 17 (2016): 1459-1466.

12. Doughty CA., et al. "Antigen receptor mediated changes in glucose metabolism in B Lymphocytes: role of phosphatidylinositol 3- kinase signaling in the glycolytic control of growth". Blood 107 (2006): 4458-4465.

13. Le A., et al. "Glucose -independent glutamine metabolism via TCA cycling for proliferation and survival in B Cells". Cell Metabolism 15 (2012): 110-121.

14. Loftus M., et al. "Amino acid -dependent c-Myc expression is essential for NK Cell metabolic and functional responses in mice". Nature Communications 9 (2018): 2341.

15. O'Neil LA and Pearce EJ. "Immunometabolism governs dendritic cell and macrophage function". Journal of Experimental Medicine 213 (2016): 15-23.

16. Robinson JM., et al. "Glycogen accumulation in polymorphonuclear leukocytes and other intracellular alterations that occur during inflammation". Journal of Cell Biology 95 (1982): 933-942.

17. Thwe PM., et al. "Cell intrinsic glycogen metabolism supports early glycolytic reprogramming required for dendritic cell immune responses". Cell Metabolism 26 (2017): 558-67. e5.

18. Lawless S., et al. "Glucose responses dendritic cell induced T cell responses". Nature Communications 8 (2017): 15620.

19. Ho PC., et al. "Phosphoenopyruvate is a metabolic checkpoint of antitumor T cell responses". Cell (2015): 1217-1218. 
20. Still ER., et al. "Hopefully devoted to Q:Targeting glutamine addiction in cancer". British Journal of Cancer 58 (2017): 533537.

21. Zhou R., et al. “[(18)F] (254R)4-Fluoroglutamine PET detects glutamine pool size changes in triple negative breast cancer in response to glutamine inhibition". Cancer Research 77 (2017): 1476-1484.

22. Cascone T., et al. "Increased tumor glycolysis characterizes immune resistance to adoptive T cell therapy". Cell Metabolism 27 (2018): 977-987.

23. Gualdoni GA., et al. "Rhinovirus induces an anabolic reprogramming in host cell metabolism essential for viral replication". Proceedings of the National Academy of Sciences of the United States of America 115 (2018): E7158-E7165.

24. Smallwood HS., et al. "Targeting metabolic reprogramming by influenza infection for therapeutic intervention". Cell Report 19 (2017): 1640-1653.

25. Chi P., et al. "Avian reovirus sigma -modulated suppression of lactate dehydrogenase and upregulation of glutaminolysis and the mTOR/eIF4E/HIF-1alpha pathway to enhance glycolysis and the TCA cycle for viral replication". Cell Microbiology 20 (2018): e12946.

26. Escoll P and Buchrieser C. "Metabolic reprogramming of host cells upon bacterial infection: why shift to Warburg -like metabolism?" FEBS Journal 285 (2018): 2146-22160.

27. Viko NP., et al. "Glycolytic dependency of high level nitric oxide resistance and virulence in Staphylococcal aureus". MBio (2015): 6 .

28. Tamune H., et al. "Cerebrospinal fluid /blood glucose ratio as an indicator for bacterial meningitis". The American Journal of Medicine 2 (2014): 263-266.

29. Cheng H., et al. "Nitric oxide in cancer metastasis". Cancer Letter 353 (2014): 1-7.

30. Mondanelli G., et al. "The immune regulation in cancer by the amino acid metabolizing enzymes ARG and IDO". Current Opinion in Pharmacology 35 (2017): 30-39.

31. Goh CC., et al. "Hepatitis C -virus induced myeloid derived suppressor cells suppresses NKCell IFN-Gamma production by altering cellular metabolism via Arginase 1". Journal of Immunology 196 (2016): 2283-2292.

32. Munn DH and Mellor AL. "IDO in the tumor microenvironment: inflammation, counter regulation, and tolerance". Trends Immunology 37 (2016): 193-207.
33. Prendergast GC., et al. "Indoleamine 2, 3-dioxygenases pathways of pathogenic inflammation and immune escape in cancer". Cancer Immunology, Immunotherapy 63 (2014): 721735 .

34. Taylor MW and Feng GS. "Relationship between interferon gamma, Indoleamine 2, 3-dioxygenases, and tryptophan catabolism". FASEB Journal 5 (1991): 2516-2522.

35. Schmidt SV and Schultz JL. "New insights into IDO biology in bacterial and viral infections". Frontier Immunology 5 (2014): 384.

36. Gobert AP., et al. "Helicobacter pylori arginase inhibits nitric oxide production by eukaryotic cells :a strategy for bacterial survival". Proceedings of the National Academy of Sciences of the United States of America 98 (2001): 13844-13849.

37. Weiberg JB., et al. "Arginine, nitric oxide, carbon monoxide, and endothelial function in severe malaria". Current Opinion in Pharmacology 21 (2008): 468-475.

38. Gerard A., et al. "Secondary T cell - Tcell synaptic interactions drive the differentiation of protective CD8+Tcells priming by dendritic cells in intact lymph nodes". Nature Immunology 14 (2013): 56-63.

39. Mingozzi F., et al. "Prolonged contact with dendritic cells turns lymph nodes-resident NK Cells into antitumor effectors". EMBO 8 (2016): 1039-1051.

40. Wensveen FM., et al. "The fourth dimensioning imuunological space: how the struggle for nutrients selects high affinity lymphocytes". Immunology Review 249 (2012): 84-103.

41. Man K., et al. "The transcription factor IRF4 is essential for TCR affinity-mediated metabolic programming and clonal expansion of T cells". Nature Immunology 14 (2013): 11551163.

42. Lin SC and Hardie DG. "AMPK: sensing glucose as well as cellular energy status". Cell Metabolism 27 (2018): 299-313.

43. Blagh J., et al. "The energy sensor AMPK regulates T cell metabolic adaptation and effector responses in vivo". Immunity 42 (2015): 41-54.

44. Ramakrishna P., et al. "Activation of the transcriptional function of the NF-kappa B protein c-Rel by O- GlcNAc glycosylation". Science Signal 6 (2016): ra75.

45. Walls J., et al. "Nutient sensing, signal transduction and immune responses". Seminar on Immunology 28 (2016): 396407. 
46. Preston GC., et al. "Single cell tuning of Myc expression by antigen receptor signal strength and interleukin -2 in T Lymphocytes". EMBO Journal 34 (2015): 2008-2024.

47. Chou C., et al. "c-Myc-induced transcription factor AP4 is required for host protection mediated byCD8+T Cells". Nature Immunology 15 (2014): 884-893.

48. Munn DH., et al. "GCN 2 kinase in T cells mediates proliferative arrest and anergy induction in response to Indoleamine 2, 3- dioxygenase". Immunity 22 (2005): 633-642.

49. Kedia -Mehta N and Finlay DK. "Competition for nutrients and its role in controlling immune responses". Nature Communications (2019): 102123.

50. Ravindran R., et al. "The amino acid sensor GCN2 controls gut inflammation by inhibiting inflassome activation". Nature 531 (2016): 523-27.

\section{Volume 3 Issue 10 October 2019}

(C) All rights are reserved by Kulvinder Kochar Kaur.,

et al. 